\title{
Changes in the Polyphenolic Profile, Carotenoids and Antioxidant Potential of Apricot (Prunus armeniaca L.) Leaves during Maturation
}

\author{
Alam Zeb *, Nadia Khadim and Waqar Ali \\ Biochemistry Laboratory, Department of Biotechnology, University of Malakand, Chakdara 18800, Pakistan; \\ nadiakhadim1902@yahoo.com (N.K.); waqarali04@yahoo.com (W.A.) \\ * Correspondence: azeb@uom.edu.pk or azebuom@gmail.com; Tel.: +92-945-761622 \\ Academic Editor: Takayuki Shibamoto \\ Received: 29 December 2016; Accepted: 23 January 2017; Published: 8 February 2017
}

\begin{abstract}
Apricot (Prunus armeniaca L.) leaves were studied to assess the potential of apricot leaves for future studies and their applications in nutraceutical and bioactive functional ingredients. The changes in the phenolic profile, carotenoids, pigments and antioxidant potential were studied at four maturation stages. Polyphenols and carotenoids were studied using reversed-phase HPLC-DAD. Pigments, total phenolic contents and radical scavenging activity were also measured. Results revealed twelve phenolic compounds in the apricot leaves. The major phenolic compounds were 3-O-caffeoylquinic acid (14.6-49.6 mg/g), 4-O-caffeoylquinic acid $(0.56-7.5 \mathrm{mg} / \mathrm{g}), 5-\mathrm{O}$-caffeoylquinic acid (5.6-25.7 mg/g) and quercetin-3-O-glucosides (8.6-19.9 mg/g), while others include caffeic acid and derivatives of coumaric acid and kaempferol. Significant changes were observed in polyphenolic compounds during maturation. Lutein $(56.7-65.7 \mu \mathrm{g} / \mathrm{g})$, neoxanthin $(0.66-4.79 \mu \mathrm{g} / \mathrm{g})$, 5,6-epoxy- $\alpha$-carotene $(5.89-7.9 \mu \mathrm{g} / \mathrm{g})$, and $\beta$-carotene $(12.3-26.9 \mu \mathrm{g} / \mathrm{g})$ were the major carotenoids. There were significant variations in the carotenoids, pigment contents, total phenolic contents and radical scavenging activity during maturation. In conclusion, significant variation occurred in the polyphenolic profile, carotenoids contents and antioxidant potential of apricot leaves under the studied conditions.
\end{abstract}

Keywords: HPLC-DAD; phenolic compounds; carotenoids; apricot leaves; pigments contents; antioxidant activity; total phenolic contents; leaves maturation

\section{Introduction}

Apricot (Prunus armeniaca L.) is one of the most important plants widely grown and popular for its characteristic fruit. Pakistan is the third major producer in the world with annual production now more than 0.5 million MT [1]. Northern areas and Khyber Pakhtunkhwa are the main cultivating areas for apricot with an annual production contribution of more than $60 \%[1,2]$. The fruit from these areas is of high quality and taste and serves as a source of economy and agriculture. Ali, Masud and Abbasi [1] studied the physico-chemical characteristics of several apricot varieties from Pakistan. These authors reported that apricot fruit contains fat $(2.1 \%-3 \%)$, proteins $(6.18 \%-8.7 \%)$, fibre $(11.85 \%-13.6 \%)$, ash $(9.45 \%-12.1 \%)$ and total sugars $(56.8 \%-64.9 \%)$ on a fresh weight basis. The reported amount of ascorbic acid was $67.39-90.94 \mathrm{mg} / 100 \mathrm{~g}$, total phenolic compounds were 4590-7310 mg GAE/100 g, total carotenoids $10.09-18.13 \mathrm{mg} / 100 \mathrm{~g} \beta$-carotene and antioxidant activity of $56.84 \%-82.33 \%$. The authors concluded that Pakistani apricots are of high nutritional quality.

Apricot is a good source of various phytochemicals, which is one of the reasons that it possess anti-microbial, anti-mutagenic, cardio-protective, hepato-protective and antioxidant activities [3]. Apricot is rich in carotenoids [4], fatty acids and sterols, volatile compounds, glycosides and 
polyphenols [3]. Polyphenols includes catechin, chlorogenic acid, cynidin-3-glucosides, epicatechin, epigallocatechin, kaempferol-3-rutinoside, neochlorogenic acid, quercetin-3-glucosides, and rutin [5]. These phenolic compounds showed variations with respect to different variety of fruits and peeling (skin). Dragovic-Uzelac, et al. [6] evaluated different apricot varieties depending on the stage of maturity and geographical regions. The authors concluded that the region of cultivation and the cultivar were two important factors that influenced the carotenoids and phenolic compounds of apricot fruits. Different phytochemicals in apricot were thus studied, which are affected by different fruit load [7]. The apricot leaves play a significant role in the development of fruit [8]. There is, however, a lack of information on the variations in the polyphenolic profile, carotenoids and antioxidant potential of apricot leaves during maturation under specific conditions. This study, therefore, had two principal objectives: (i) to determine the important polyphenolic composition, carotenoids and antioxidant potential of the apricot leaves for potential biological applications and (ii) their variations during maturation that may have potential importance for fruit development.

\section{Materials and Methods}

\subsection{Materials}

Caftaric acid, quercetin-3-glucoside, 3-O-caffeoylquinic acid, 4-O-caffeoylquinic acid, 5-O-caffeoylquinic acid coumaric acid, lutein and $\beta$-carotene, DPPH \& methanol were purchased from Sigma-Aldrich (Steinheim, Germany), while caffeic acid was purchased from TCI (Tokyo, Japan). Glacial acetic acid was purchased from Daejung Chemicals (Namyangju, Korea). Ultrapure deionized double distilled water with less than $5 \mathrm{~m} \Omega$ was used. All other chemicals and reagents were of analytical standard of high purity.

\subsection{Sample Collection and Preparation}

Indigenous apricot leaves were collected from the apricot fields in Thana, District Malakand, Khyber Pakhtunkhwa irrigated with fresh water. The samples were collected four times for 2 months with an interval of fifteen days (7 March to 21 April). The temperature during each collection was $14.9,18.4,21.3$ and $25.7^{\circ} \mathrm{C}$. The total sunshine was $11 \mathrm{~h}$ and $40 \mathrm{~min}, 11 \mathrm{~h}$ and $47 \mathrm{~min}, 12 \mathrm{~h}$ and $46 \mathrm{~min}$ and $13 \mathrm{~h}$ and $45 \mathrm{~min}$ during the 1st, $2 \mathrm{nd}, 3 \mathrm{rd}$ and 4 th collections, respectively. The samples were collected from the stage of sprouting ( 20 February) to mature leaves. Since leaves mature with respect to time from sprouting to full sized leaves before fruit development, the specific interval (one week) was considered as the maturation stage. Apricot leaves were shade dried after collection. When the samples were completely dried, they were crushed and ground to a fine powder. The leaf extracts were prepared from the powdered samples.

\subsection{Sample Preparation}

Extraction of phenolic compounds was carried out using methanol and water $(5: 5, v / v)$ as a solvent. For this purpose, a powdered sample of $1 \mathrm{~g}$ was mixed with a methanol-water mixture $(25 \mathrm{~mL})$. The resultant mixture was kept at $70{ }^{\circ} \mathrm{C}$ for one hour in a water bath and shaken vigorously. A filtered extract was obtained after $30 \mathrm{~min}$ of centrifugation at $4000 \mathrm{rpm}$. The filtered extract $(2 \mathrm{~mL})$ was filtered using Agilent PFTE filers $(0.45 \mu \mathrm{m})$ and transferred into $2 \mathrm{~mL}$ HPLC vials. Carotenoids were extracted using cold acetone, followed by saponification, partitioning into aqueous and hexane layers and evaporation with nitrogen under ambient conditions and daylight [9].

\subsection{Chromatography of Phenolic Compounds}

The phenolic profile was determined using an Agilent 1260 HPLC system, consisting of a quaternary pump, degasser, auto-sampler and diode array detector (DAD). The separation was carried out using an Agilent rapid resolution column (Zorbax Eclipse C18, $406 \times 250 \mathrm{~mm}, 5 \mu \mathrm{m}$ ). The gradient system used in this study consisted of methanol-acetic acid-deionized water (10:2:88, 
$v / v)$ as solvent $A$, while the solvent $B$ composition was methanol-acetic acid-deionized water $(90: 2: 8$, $v / v$ ) at a flow rate of $1 \mathrm{~mL} / \mathrm{min}$. The binary gradient program was started with $100 \% \mathrm{~A}$ at $0 \mathrm{~min}, 85 \%$ $\mathrm{A}$ at $5 \mathrm{~min}, 50 \% \mathrm{~A}$ at $20 \mathrm{~min}, 30 \% \mathrm{~A}$ at $25 \mathrm{~min}$ [10]. The separation was achieved within $30 \mathrm{~min}$ of elution. The DAD detector was set to $320 \mathrm{~nm}$ for analyses of phenolic compounds, while the spectra were recorded in the range of 190 to $600 \mathrm{~nm}$. The identification of phenolic compounds was based on either the available standards (caftaric acid, quercetin-3-glucoside, three caffeoyl quinic acids, caffeic acid, coumaric acid), their retention times and absorption spectra, or by comparing with absorption spectra reported in the scientific literature. The quantification of identified compounds was based on the standard calibration curves (five points in duplicates) of the standard compounds and is expressed as $\mathrm{mg} / \mathrm{g}$ of dry weight (DW) of the sample.

\subsection{Chromatography of Carotenoids}

Carotenoids were determined using a reversed-phase HPLC system. The separation was carried out using an Agilent rapid resolution column (Zorbax Eclipse C18, $4.6 \times 100 \mathrm{~mm}, 3.5 \mu \mathrm{m}$ ). The gradient system consisted of solvent A (methanol: deionized water, 92:8, $v / v$, with $10 \mathrm{mM}$ Ammonium acetate), solvent B (deionized water containing $0.01 \mathrm{mM}$ ) and solvent C as MTBE $(100 \%)$ with a flow rate of $1 \mathrm{~mL} / \mathrm{min}$. The tertiary gradient program was started with $80 \%: 18 \%: 2 \%$ of A:B:C at $0 \mathrm{~min}$ and reached $60 \%: 0 \%: 40 \%$ at $40 \mathrm{~min}$ [11]. The spectra were recorded in the range of 190 to $600 \mathrm{~nm}$ and the chromatograms were obtained at $450 \mathrm{~nm}$. The identification of carotenoids was based on either the available standards ( $\beta$-carotene \& lutein), their retention times, or absorption spectra reported in the literature [12]. The quantification of identified compounds was based on peak area and is expressed as $\mu \mathrm{g} / \mathrm{g}$ dry weight (DW) of the sample.

\subsection{Determination of Pigments Contents}

Chlorophyll $a \& b$, and total chlorophyll contents were determined using a method reported previously using the Pharmaspec 1700 spectrophotometer (Shimadzu, Kyoto, Japan) [13]. The extracts of powdered samples were dissolved in methanol-water and the absorbance was measured at 645 and $663 \mathrm{~nm}$. The values are expressed as $\mathrm{mg} / \mathrm{mL}$.

\subsection{Determination of Total Phenolic Contents}

Total phenolic contents (TPC) of apricot leaves extracts were determined using Folin-Ciocalteu reagent as recently described in detail [14]. The FC reagent was prepared fresh in the laboratory. Gallic acid was used to prepare a standard calibration curve (six points in triplicate). The concentration range of the calibration was from 5 to $100 \mathrm{mg}$. TPC was measured using a spectrophotometer and expressed as $\mathrm{mg}$ of gallic acid equivalents (GAE) per $100 \mathrm{~g}$ of the sample.

\subsection{Radical Scavenging Activity}

The radical scavenging activity (RSA) of each sample in triplicate was determined using the DPPH method [15]. Fresh DPPH solution $(0.1 \mathrm{mM})$ was prepared in methanol every day. For measurement, DPPH solution $(1.90 \mathrm{~mL})$ was mixed with methanolic extract $(10 \mu \mathrm{L})$ at a concentration of $1.01 \mathrm{mg} / \mathrm{mL}$ with the leaves. The resultant mixture was placed in a dark chamber for $30 \mathrm{~min}$. Then absorbance of the sample was measured using a Pharmaspec 1700 spectrophotometer (Shimadzu, Japan) at $515 \mathrm{~nm}$. The RSA of the samples was calculated using the given equation: \% RSA = (Ac - As) $/ \mathrm{Ac}$, where, Ac is the absorbance of the control and As is the absorbance of the apricot leaf extract.

\subsection{Data Analyses}

All samples were measured in replicate $(n=3)$. Data were analysed for variation using one-way analysis of variance (ANOVA) and Tukey's method using Graph Pad Prism 5 for Windows version 5.03 (Graph Pad Software Inc., La Jolla, CA, USA, 2009). 


\section{Results}

\subsection{Phenolic Compounds in Apricot Leaves}

Figure 1A-D shows the HPLC-DAD chromatograms of the apricot leaves at the different maturation stages. A total of ten polyphenolic compounds was identified. The identification details of each compound represented by a peak number in the chromatograms are shown in Table 1. Compound 1 was identified as 3-O-caffeoylquinic acid (chlorogenic acid) with $\lambda_{\max }$ of 234, 298sh and $325 \mathrm{~nm}$ by comparing with a standard compound. Compound 2, which was eluted at 5.2 min, was coumaric acid derivative, with $\lambda_{\max }$ of 283, 288 and $314 \mathrm{~nm}$. Compound 3 was identified as 5-O-caffeoylquinic acid (neo-chlorogenic acid) with $\lambda_{\max }$ of 236, $298 \mathrm{sh}$ and $326 \mathrm{~nm}$. This compound was eluted at $5.9 \mathrm{~min}$. At 6.1, 6.3 and 6.6 min of elution, coumaroyl hexose (4), caffeic acid (5) and 4-O-caffeoylquinic acid (6) were identified. Hydroxyferuloylglucose was identified at the retention time of $7.1 \mathrm{~min}$ with $\lambda_{\max }$ of 240, 305 and $326 \mathrm{~nm}$ as compound 7. No other compounds were identified until $14 \mathrm{~min}$, when quercetin-3-glucoside was eluted with characteristic $\lambda_{\max }$ of 256 and $354 \mathrm{~nm}$ and marked as compound 8 based on a comparison with the standard compound. At retention times of 16.7 and $17.2 \mathrm{~min}$, kaempferol hexoside (compound 9) with characteristic $\lambda_{\max }$ of 285, 325 and $346 \mathrm{~nm}$ and kaempferol derivative (compound 10) were eluted. Compound 10 had $\lambda_{\max }$ of 285, 324 and $345 \mathrm{~nm}$. However, the HPLC-DAD method was not sufficient to correctly identify the derived component of kaempferol. Two more compounds, i.e., coumaric acid and caftaric acid, were identified at the stage of 45 days of maturation and were labelled as compound 11 and 12, respectively. However, these compounds were not identified in any other maturation stages.

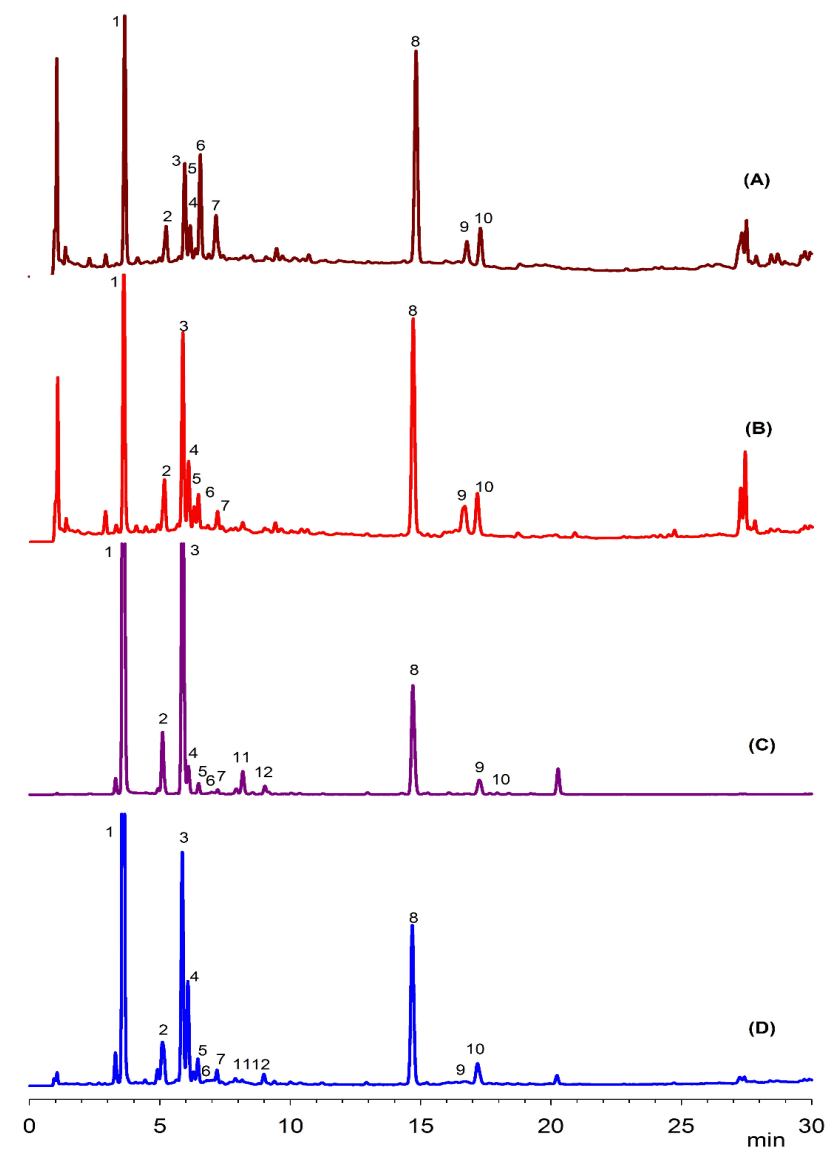

Figure 1. Representative HPLC-DAD chromatograms of the apricot leaves during maturation at $320 \mathrm{~nm}$. (A) 15 days; (B) 30 days; (C) 45 days; and (D) 60 days. The identification of the peaks are given in Table 1. 
Table 1. Identification of polyphenolic compounds in apricot leaf samples.

\begin{tabular}{lcccc}
\hline Peak & Rt (min) & Tentative Identification & $\begin{array}{c}\text { HPLC-DAD } \\
\boldsymbol{\lambda}_{\max }(\mathbf{n m})\end{array}$ & Identification \\
\hline 1 & 3.6 & 3-O-Caffeoylquinic acid & $234,298 \mathrm{sh}, 325$ & Standard \\
2 & 5.2 & p-Coumaric acid derivative & $283,288,314$ & {$[16]$} \\
3 & 5.9 & 5-O-Caffeoylquinic acid & $236,298 \mathrm{sh}, 326$ & Standard \\
4 & 6.1 & p-Coumaroyl hexose & 285,316 & {$[16]$} \\
5 & 6.3 & Caffeic acid & $232,302,326$ & Standard \\
6 & 6.6 & 4-Caffeoylquinic acid & $232,303,326$ & Standard \\
7 & 7.1 & Hydroxy feruloyl glucose & $240,305,326$ & [15] \\
8 & 14.8 & Quercetin-3-glucoside & 256,354 & Standard \\
9 & 16.7 & Kaempferol hexoside & $285,325,346$ & [17] \\
10 & 17.2 & Kaempferol derivative & $285,324,345$ & [17] \\
11 & 8.1 & Coumaric acid & 230,312 & Standard \\
12 & 9.2 & Caftaric acid & $245,298 \mathrm{sh}, 328$ & Standard \\
\hline
\end{tabular}

\subsection{Changes in the Phenolic Compounds}

Figure 2 shows the changes in the phenolic compounds during the maturation of apricot leaves. Chlorogenic acid (3-O-caffeoylquinic acid) was present at the highest level $(15.67 \mathrm{mg} / \mathrm{g}$ ) at 15 days of maturation, and reached significantly $(p<0.01)$ higher values $(48.76$ and $49.6 \mathrm{mg} / \mathrm{g})$ as shown in Figure 2A. Quercetin-3-glucoside $(19.69 \mathrm{mg} / \mathrm{g})$ was present in the second highest amount. There were no significant $(p>0.01)$ changes during 15 and 30 days of maturation, while a significant decrease was observed at 45 days. No significant changes were observed at 60 days of maturation. Thus, a high correlation $\left(R^{2}=0.9114\right)$ of these two compounds was observed during the selected maturation stages.
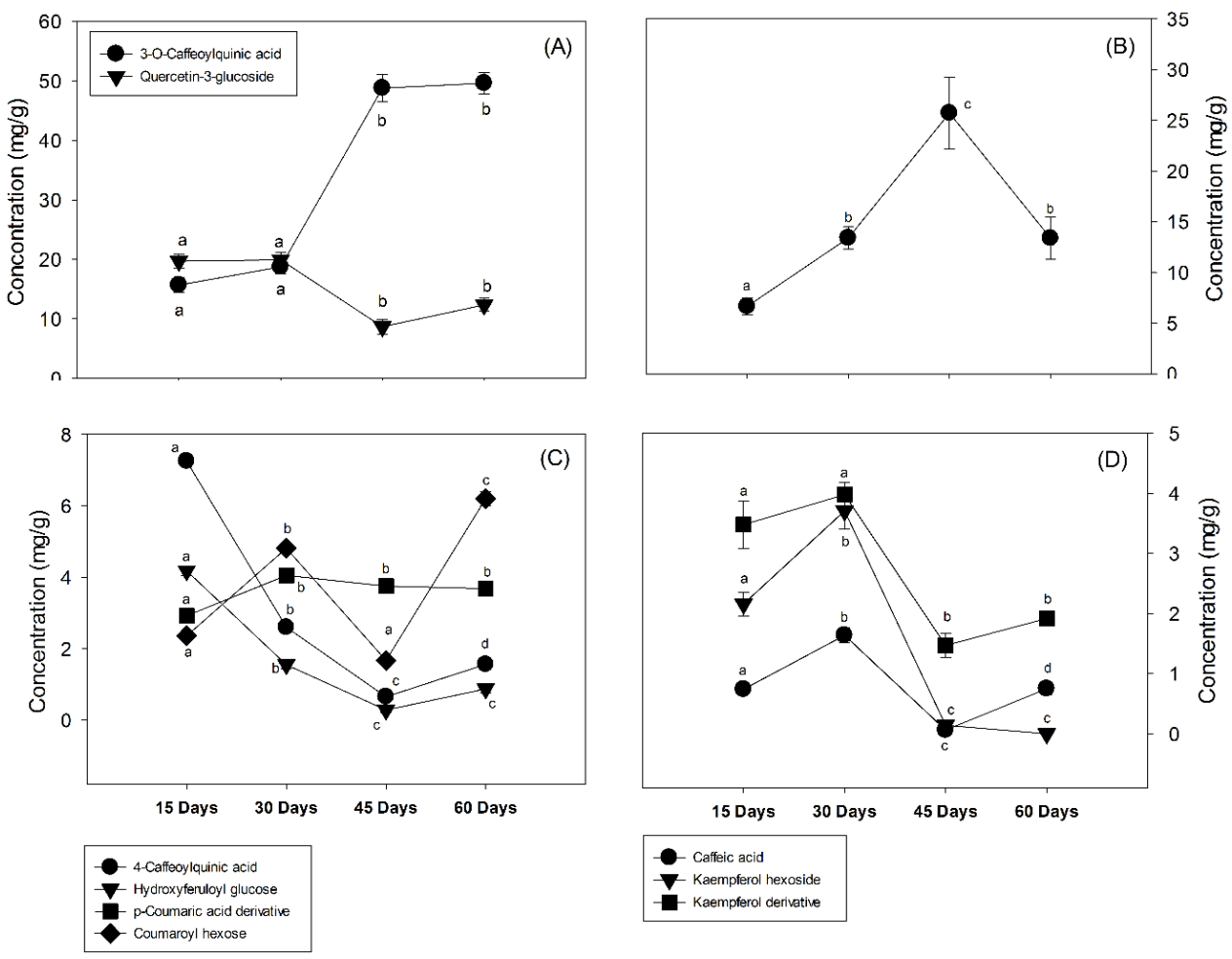

Figure 2. Changes in the polyphenolic compounds of apricot leaves during maturation. (A) 3-O-Caffeoylquinic acid, quercetin-3-glucoside; (B) 5-O-Caffeoylquinic acid; (C) 4-O-Caffeoylquinic acid, hydroxyferuloyl glucose, p-coumaric acid derivative; and (D) caffeic acid, kaempferol hexoside, and laempferol derivative. Significant levels are mentioned in the text. 
Compound 3 was tentatively identified as 5-O-caffeoylquinic acid (neo-chlorogenic acid), which was the third most abundant compound in terms of the percent amount present in the apricot leaves at different stages (Figure 2B). Significant $(p<0.05)$ increases occurred during the first three stages of maturation, while a significant decrease occurred in the amount of 5-O-caffeoylquinic acid at 60 days of maturation. The amount of 5-O-caffeoylquinic acid at 60 days was the same as that at 30 days of maturation. Figure $2 \mathrm{C}$ shows that the amount of 4-O-caffeoylquinic acid decreased significantly with maturation. A similar trend was observed in the case of hydroxyferuloyl glucose, the amount of which was half of the amount of 4-O-caffeoylquinic acid. The decrease in these two compounds was significantly correlated $\left(R^{2}=0.999\right)$ with each other. The decrease in the amount of 4 -O-caffeoylquinic acid was highly correlated $\left(R^{2}=0.6794\right)$ with the increase in the amount of 5 -O-caffeolyquinic acid. A significant $(p<0.05)$ increase was observed in the amount of $p$-coumaric acid derivatives at 30 days of maturation, while no significant variation was observed further as shown in Figure 2C. The amount of coumaroyl hexose significantly $(p<0.05)$ increased at 30 days of maturation, while at 45 days, a significant decrease was observed. A further significant $(p<0.05)$ increase was observed at 60 days of maturation. Similar trends were observed in the amount of caffeic acid, kaempferol hexoside and kaempferol derivatives as shown in the Figure 2D.

\subsection{Carotenoid Contents}

Figure 3 shows the typical chromatogram of carotenoid contents in the apricot leaves. Using the available standards and absorption spectra in the reported literature, ten carotenoids were identified. These contained two isomers of neoxanthin (all-trans-neoxanthin \& 9-cis-neoxanthin), cis- $\gamma$-carotene, all-trans-violaxanthin, all-trans-lutein, three isomers of $\alpha$-carotene (5,6-epoxy- $\alpha$-carotene, 15 -cis- $\alpha$-carotene and 13-cis- $\alpha$-carotene) and $\beta$-carotene and its 9-cis isomer as shown in the Table 2. These results showed that $\alpha-, \beta$-carotene and lutein were present in high amounts in all apricot leaves.

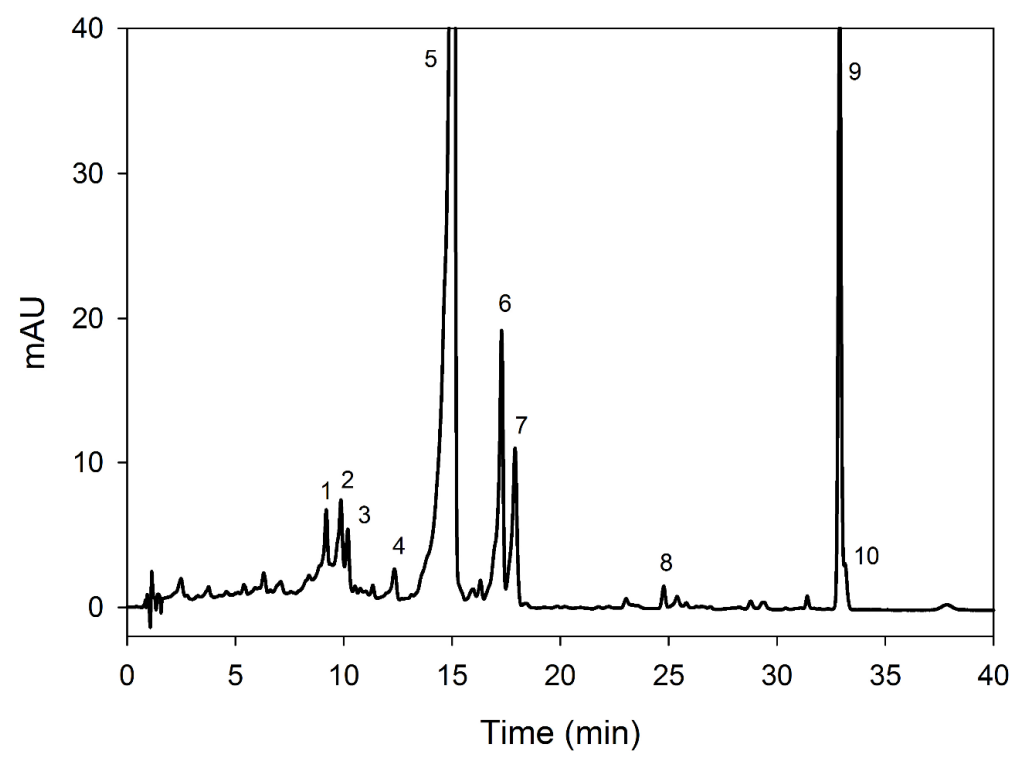

Figure 3. Representative HPLC-DAD chromatograms at $450 \mathrm{~nm}$ of carotenoids of the apricot leaves. Numbers above the peaks represent individual compounds with details given in Table 2. 
Table 2. Identification and changes in the Carotenoid contents of Apricot leaf samples. Identification was based on comparison with the absorption spectra of the standard compounds and data reported in the literature [12].

\begin{tabular}{|c|c|c|c|c|c|c|c|}
\hline \multirow{2}{*}{ Peak } & \multirow{2}{*}{$R_{\mathrm{t}}(\min )$} & \multirow{2}{*}{$\begin{array}{c}\text { Tentative } \\
\text { Identification }\end{array}$} & \multirow{2}{*}{$\begin{array}{c}\text { HPLC-DAD } \\
\lambda_{\max }(\mathrm{nm})\end{array}$} & \multicolumn{4}{|c|}{ Carotenoids $(\mu \mathrm{g} / \mathrm{g})$} \\
\hline & & & & 15 & 30 & 45 & 60 \\
\hline 1 & 9.1 & All-trans-neoxanthin & $468,444,420$ & $2.01 \pm 0.1^{\mathrm{a}}$ & $4.79 \pm 0.3^{b}$ & $0.66 \pm 0.1^{\mathrm{c}}$ & $4.01 \pm 0.3^{b}$ \\
\hline 2 & 9.8 & 9-Cis-neoxanthin & $464,436,418$ & $2.46 \pm 0.2^{\mathrm{a}}$ & $3.6 \pm 0.14^{b}$ & $1.01 \pm 0.2^{\mathrm{c}}$ & $2.34 \pm 0.3^{\mathrm{a}}$ \\
\hline 3 & 10.1 & Cis- $\gamma$-carotene & $464,438,422$ & $1.18 \pm 0.1^{\mathrm{a}}$ & $1.55 \pm 0.1^{\mathrm{a}}$ & $1.33 \pm 0.1^{\mathrm{a}}$ & $2.34 \pm 0.1^{b}$ \\
\hline 4 & 12.3 & All-trans-violaxanthin & $472,444,420,330$ & $0.54 \pm 0.02^{\mathrm{a}}$ & $0.42 \pm 0.01^{\mathrm{a}}$ & $0.27 \pm 0.1^{\mathrm{b}}$ & $0.26 \pm 0.1^{b}$ \\
\hline 5 & 15.0 & All-trans-lutein & $474,446,422$ & $65.7 \pm 1.4^{\mathrm{a}}$ & $61.1 \pm 0.9^{b}$ & $58.2 \pm 0.4^{\mathrm{c}}$ & $56.7 \pm 0.8^{d}$ \\
\hline 6 & 17.2 & 5,6-epoxy- $\alpha$-carotene & $468,440,420$ & $7.78 \pm 0.5^{\mathrm{a}}$ & $7.9 \pm 0.3^{\mathrm{a}}$ & $5.89 \pm 0.2^{b}$ & $6.41 \pm 0.7^{c}$ \\
\hline 7 & 17.9 & 15 -Cis- $\alpha$-carotene & $466,440,420,330$ & $4.84 \pm 0.3^{\mathrm{a}}$ & $4.69 \pm 0.2^{\mathrm{a}}$ & $2.0 \pm 0.2^{b}$ & $4.14 \pm 0.1^{\mathrm{a}}$ \\
\hline 8 & 24.7 & 13 -Cis- $\alpha$-carotene & $466,440,420$ & $0.39 \pm 0.02^{a}$ & $0.32 \pm 0.02^{\mathrm{a}}$ & $0.62 \pm 0.06^{b}$ & $0.21 \pm 0.03^{c}$ \\
\hline 9 & 32.8 & $\beta$-Carotene & 476,450 & $12.3 \pm 0.1^{\mathrm{a}}$ & $13.6 \pm 0.2^{\mathrm{a}}$ & $26.9 \pm 1.1^{b}$ & $18.5 \pm 2.1^{\mathrm{c}}$ \\
\hline 10 & 33.2 & 9-Cis- $\beta$-carotene & $468,446,335$ & $0.81 \pm 0.04^{\mathrm{a}}$ & $1.21 \pm 0.06^{b}$ & $2.2 \pm 0.1^{\mathrm{c}}$ & $1.13 \pm 0.2^{b}$ \\
\hline
\end{tabular}

Data are presented as the mean with standard deviation $(n=3)$. Different letters ${ }^{(\mathrm{a}-\mathrm{d})}$ represent significance at $p<0.05$ (Tukey's test).

\subsection{Changes in Carotenoid Contents}

Table 2 shows that a significant increase $(p<0.05)$ occurred in the amounts of all-trans-neoxanthin and its isomer with increasing maturation time. However, a significant loss in these carotenoids was observed at 45 days. No significant changes were observed in the amounts of cis- $\gamma$-carotene and all-trans-violaxanthin, except at 60 days, the amount of these carotenoids were higher or lower. A significant $(p<0.05)$ decrease occurred in the amount of all-trans-lutein from 65.7 to $56.6 \mu \mathrm{g} / \mathrm{g}$. There was no significant changes in the amounts of $\alpha$-carotene and its isomers at 15 and 30 days of maturation, while significant changes were observed at 45 and 60 days of maturation. Similarly, $\beta$-carotene was present in the second highest amount $(12.3 \mu \mathrm{g} / \mathrm{g})$. There was no significant change during 15-30 days, while a significant increase $(p<0.05)$ occurred at 45 days of maturation. The amount was less than at 45 days and higher than at 15 and 30 days of maturation. There was a significant increase in the amount of 9-cis- $\beta$-carotene with an increase in maturation; however, the amount decreased to the original value measured at 30 days. These results showed that significant changes occurred with maturation of the apricot leaves.

\subsection{Changes in Pigment Contents}

Figure 4 shows the changes in the chlorophyll contents of the apricot leaves. Chlorophyll $a$ was higher than chlorophyll $b$. It was observed that chlorophyll $a$ decreased significantly $(p<0.01)$ to $5.40 \mathrm{mg} / \mathrm{mL}$ with maturation until 45 days from the $23.37 \mathrm{mg} / \mathrm{mL}$ level measured at 15 days. The values increased to $19.90 \mathrm{mg} / \mathrm{mL}$ at 60 days of maturation. Similar observations was also reported for chlorophyll $b$, where values of $13.18,8.78,6.22 \mathrm{mg} / \mathrm{mL}$ were obtained for 15,30 and 45 days of maturation, respectively. This shows a significant $(p<0.01)$ decrease in chlorophyll $b$. The value increased by 60 days of maturation. Total chlorophyll showed a similar trend of variation as that described above for chlorophyll $a \& b$. 


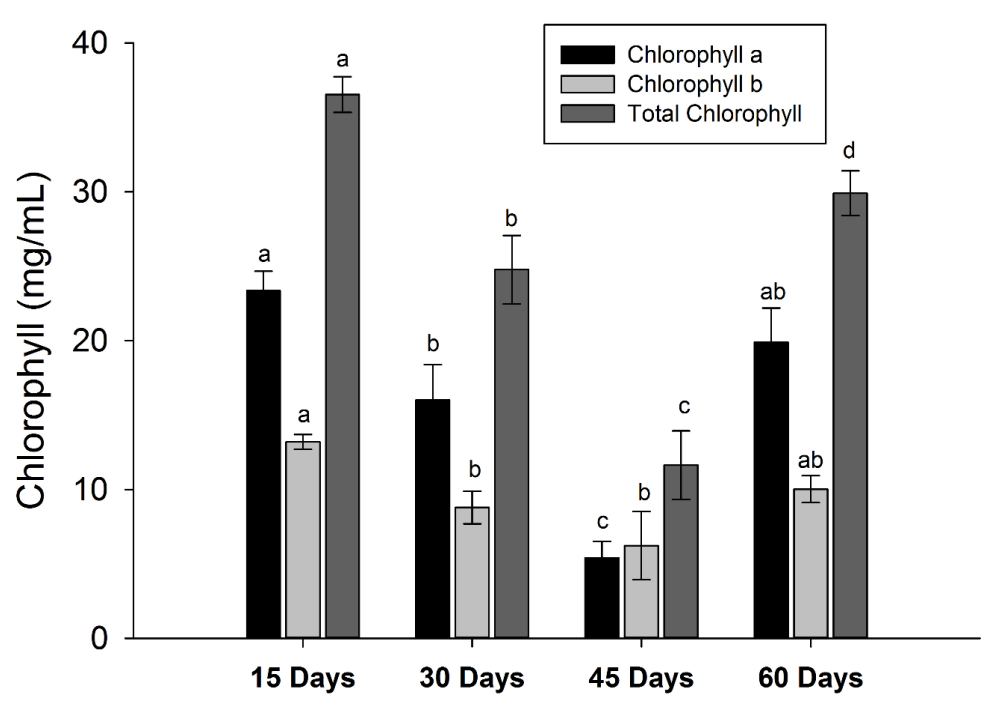

Figure 4. Changes in the pigment contents of the apricot leaves during maturation. Different letters (a-d) in each column represent significant differences at $p<0.01$ (Tukey's test).

\subsection{Changes in TPC and Radical Scavenging Activity}

Figure 5A shows the TPC values of the apricot leaves during maturation. There was a significant $(p<0.05)$ increase in the TPC from 15 to 45 days of maturation. There was no significant variation between 30 and 45 days of maturation. These results show that total phenolic contents increased with maturation. Figure 5B shows the RSA values of the apricot leaves during maturation. There was a significant $(p<0.05)$ decrease in the RSA at 15 and 30 days of maturation. A significant increase was then observed at 45 days and 60 days. The \% RSA values at 30 days were similar to those of $0.5 \mathrm{mg} / \mathrm{mL}$ of BHT, while the amounts at 15,45 and 60 days were in the range of 0.5 to $1.5 \mathrm{mg} / \mathrm{mL}$ BHT equivalent \% RSA. There was no significant variation in the RSA values at 45 and 60 days of maturation. The results showed that the RSA values increased with maturation. The HPLC data of the phenolic compounds were compared with RSA values during each maturation stage. It was found that only three compounds had a strong correlation with the RSA values, i.e., 3-O-caffeoylquinic acid, kaempferol derivative and quercetin-3-glucoside. A strong positive correlation coefficient $\left(R^{2}=0.8224\right)$ was found between RSA and 3-O-caffeoylquinic acid, while strong negative correlations of $R^{2}=0.9526$ and $R^{2}=0.8406$ were observed for the kaempferol derivative and quercetin-3-glucoside.

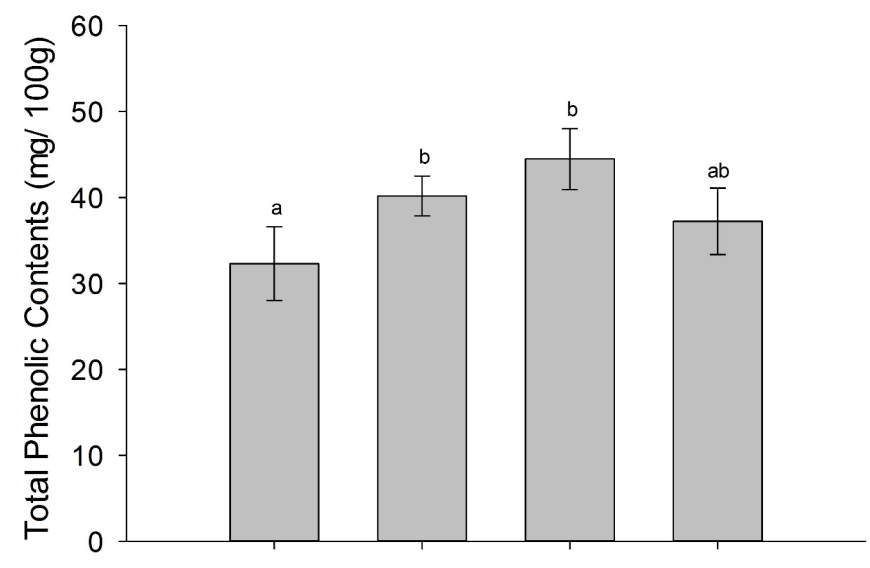

(A)

Figure 5. Cont. 


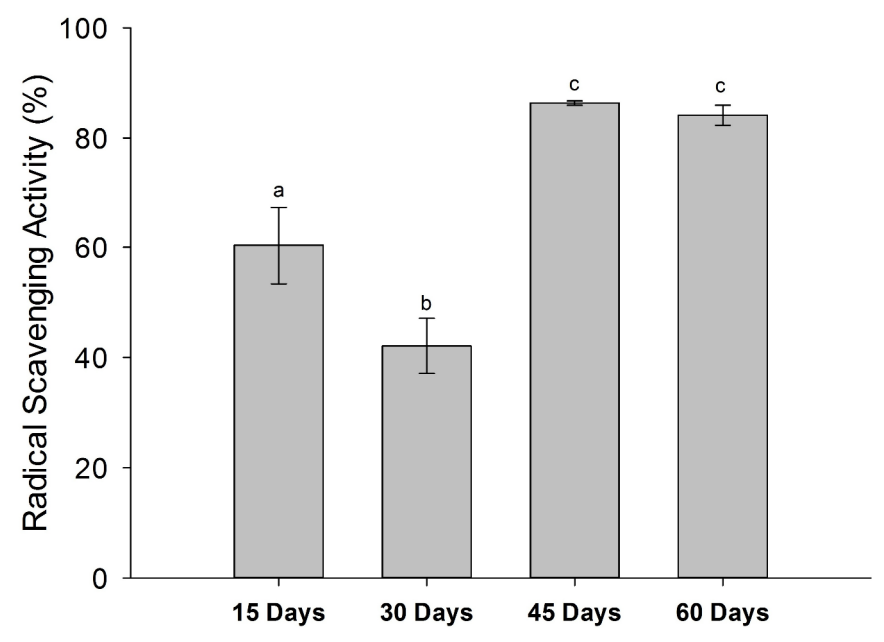

(B)

Figure 5. (A) Total Phenolic Contents (mg/100 g); (B) Radical Scavenging Activity (\%). Changes in the total phenolic contents and radical scavenging activity of the apricot leaves during maturation. Different letters (a-e) in each column represent significant differences at $p<0.05$ (Tukey's test).

\section{Discussion}

Apricot leaves are rich in various phenolic compounds as shown in Figure 1. The present results showed high amounts of caffeoyl quinic acids such as 3-O-caffeoylquinic acid, 4-O-caffeoylquinic acid (crypto-chlorogenic acid) and 5-O-caffeoylquinic acid (neo-chlorogenic acid). These compounds were identified from authentic standards and previous reports [16-18]. Neo-chlorogenic acid and chlorogenic acid were previously identified in apricot peel extract [19], and unpeeled and peeled canned apricot fruit [20]. Similarly, Dragovic-Uzelac, et al. [21] showed that caffeoyl quinic acids were the dominant polyphenols during apricot fruit ripening. This suggests that caffeoyl quinic acids are a class of compounds that are present in various products of Apricot and can thus be used as a marker for variations or identification of food products. The increase in the chlorogenic acid in the apricot leaves may be due to the heat stress, which increased during maturation [22]. Coumaric acid and coumaroyl hexose were identified in relatively small amounts. Coumaric acid was identified in apricot fruit [21] and has strong antioxidant potential. Similarly, caffeic acid, which was determined using a standard compound, was previously identified in raw and puree apricot cultivars [21].

Kaempferol derivatives were identified in the apricot leaves in this study and have been identified in apricot fruit by several authors [6,20,21]. Quercetin-3-O-glucoside is an important glycoside formed from quercetin and is an important component of apricot fruit [23]. This compound was identified in apricot fruit by several authors [5,6]. Campbell, Merwin and Padilla-Zakour [5] showed that maturity had no effect on the quercetin-3-glucoside contents of different varieties of apricot fruits. The present results show a significant decrease in quercetin-3-glucoside in the apricot leaves during maturation. This decrease may be due to the heat stress, which increased during the leaf maturation [22]. Studies have shown that quercetin-3-O-glucoside possesses strong antioxidant effects [24]. The oral supplementation of quercetin-3-O-glucoside \& queretin showed that glucoside was well absorbed by the intestinal system of the rats compared with quercetin alone [25]. These results suggest that apricot leaves contain important polyphenolic compounds, which have potential biological importance. The changes in the polyphenolic compounds in the leaves during maturation may be correlated with fruit development and thus should be considered in apricot agriculture. Variation in these compounds may occur due to the variety, climate, soil and environmental conditions. The exact structures of each derivative compounds in this study can be determined using mass spectrometry.

Apricot leaves are rich in lutein, $\alpha$ - and $\beta$-carotenes. These carotenoids are among the major carotenoids in the apricot leaves and fruits [3,26]. Katayama, et al. [27] showed that violaxanthin and 
$\beta$-carotene increased during the maturation of apricot fruits, while this study showed that the amount of these carotenoids decreased in the first two stages of maturation. This may be due to the transport or transformations of these carotenoids to other carotenoids in the fruit. These authors also reported that lutein decreased with maturation, which might be related to its lower level in the leaves. These results indicate that important carotenoids were present in the apricot leaves and changes in these compounds were observed during maturation, which might be important for fruit development.

Pigments such as chlorophylls play a key role in the photosynthesis and defence of the plant against external stress. In the present study, chlorophylls decreased significantly with maturation until 45 days. Sabir et al. [28] showed that the chlorophyll content of young leaves had a high correlation with iron concentration, which is an indicator of the vital role of iron in the chlorophyll content of leaves.

The total phenolic content (TPC) of the apricot leaves increased with maturation as shown in Figure 4A. The increase was highly correlated with the increase or decrease in the polyphenolic profile of the leaves. Phenolic compounds have high antioxidant activity as indicated by the high correlation coefficient and RSA values. The present results showed that RSA values increased with maturation and each maturation stage was known for its increase in heat stress. These results are in agreement with the results of Madrau, Piscopo, Sanguinetti, Del Caro, Poiana, Romeo and Piga [21], who showed that heat stress increased the antioxidant activity of the apricot fruits. These results indicate that apricot leaves have high amounts of phenolic compounds and important carotenoids, with high levels of antioxidant activity and thus could be used as a potential biological source of natural antioxidant substances.

\section{Conclusions}

In conclusion, apricot leaves were collected at four maturation stages. HPLC-DAD analyses revealed twelve polyphenolic compounds in the apricot leaves. The major phenolic compounds were 3-O-caffeoylquinic acid, 4-O-caffeoylquinic acid, 5-O-caffeoylquinic acid and quercetin-3-O-glucosides. Other phenolic compounds included coumaric acid or its derivatives, kaempferol or its derivatives, and caffeic acid. Significant changes were observed in the polyphenolic compounds during maturation. Lutein, neoxanthin, $\alpha$-, and $\beta$-carotenes were the major carotenoids. There were significant variations in the carotenoids, pigment contents, total phenolic contents and radical scavenging activity during maturation. In conclusion, apricot leaves are a good source of polyphenolic compounds \& carotenoids and the changes in these compounds may affect fruit development and thus should be considered for the best agriculture practice of this plant.

Acknowledgments: The work was carried out under NRPU project No. 2344 and therefore, the Higher Education Commission of Pakistan is greatly acknowledged.

Author Contributions: A.Z. and W.A. designed the experiments, N.K. \& A.Z. carried out the experiments, A.Z. wrote the manuscript. All authors contributed equally to this work and agree upon this final version of manuscript.

Conflicts of Interest: The authors declare no conflict of interest.

\section{Abbreviations}

The following abbreviations are used in this manuscript:

HPLC-DAD High performance liquid chromatography with diode array detection

TPC Total phenolic contents

RSA Radical scavenging activity

GAE Gallic acid equivalent

DPPH Diphenylpicryl hydrazine radical

ANOVA Analysis of variance 


\section{References}

1. Ali, S.; Masud, T.; Abbasi, K.S. Physico-chemical characteristics of apricot (Prunus armeniaca L.) grown in northern areas of Pakistan. Sci. Hortic. 2011, 130, 386-392. [CrossRef]

2. Ali, S.; Masud, T.; Abbasi, K.S.; Mahmood, T.; Ali, A. Some physico-chemical and functional attributes of six indigenous apricot genotypes from Gilgit-Baltistan, Pakistan. Int. J. Biosci. 2014, 4, 221-231.

3. Erdogan-Orhan, I.; Kartal, M. Insights into research on phytochemistry and biological activities of Prunus armeniaca L. (apricot). Food Res. Int. 2011, 44, 1238-1243. [CrossRef]

4. Zaghdoudi, K.; Pontvianne, S.; Framboisier, X.; Achard, M.; Kudaibergenova, R.; Ayadi-Trabelsi, M.; Kalthoum-cherif, J.; Vanderesse, R.; Frochot, C.; Guiavarc'h, Y. Accelerated solvent extraction of carotenoids from: Tunisian kaki (Diospyros kaki L.), peach (Prunus persica L.) and apricot (Prunus armeniaca L.). Food Chem. 2015, 184, 131-139. [CrossRef] [PubMed]

5. Campbell, O.E.; Merwin, I.A.; Padilla-Zakour, O.I. Characterization and the effect of maturity at harvest on the phenolic and carotenoid content of northeast USA apricot (Prunus armeniaca) varieties. J. Agric. Food Chem. 2013, 61, 12700-12710. [CrossRef] [PubMed]

6. Dragovic-Uzelac, V.; Levaj, B.; Mrkic, V.; Bursac, D.; Boras, M. The content of polyphenols and carotenoids in three apricot cultivars depending on stage of maturity and geographical region. Food Chem. 2007, 102, 966-975. [CrossRef]

7. Roussos, P.A.; Sefferou, V.; Denaxa, N.-K.; Tsantili, E.; Stathis, V. Apricot (Prunus armeniaca L.) fruit quality attributes and phytochemicals under different crop load. Sci. Hortic. 2011, 129, 472-478. [CrossRef]

8. Wang, H.; Petri, C.; Burgos, L.; Alburquerque, N. Efficient in vitro shoot regeneration from mature apricot (Prunus armeniaca L.) cotyledons. Sci. Hortic. 2013, 160, 300-305. [CrossRef]

9. Azevedo-Meleiro, C.H.; Rodriguez-Amaya, D.B. Qualitative and quantitative differences in carotenoid composition among Cucurbita moschata, Cucurbita maxima, and Cucurbita pepo. J. Agric. Food Chem. 2007, 55, 4027-4033. [CrossRef] [PubMed]

10. Zeb, A. A reversed phase HPLC-DAD method for the determination of phenolic compounds in plant leaves. Anal. Methods 2015, 7, 7753-7757. [CrossRef]

11. Zeb, A. A simple, sensitive HPLC-DAD method for simultaneous determination of carotenoids, chlorophylls and $\alpha$-tocopherol leafy vegetables. J. Food Meas. Charact. 2016. [CrossRef]

12. De Rosso, V.V.; Mercadante, A.Z. Identification and quantification of carotenoids, by HPLC-PDA-MS/MS, from Amazonian fruits. J. Agric. Food Chem. 2007, 55, 5062-5072. [CrossRef] [PubMed]

13. Barros, L.; Carvalho, A.M.; Morais, J.S.; Ferreira, I.C.F.R. Strawberry-tree, blackthorn and rose fruits: Detailed characterisation in nutrients and phytochemicals with antioxidant properties. Food Chem. 2010, 120, 247-254. [CrossRef]

14. Prior, R.L.; Wu, X.; Schaich, K. Standardized methods for the determination of antioxidant capacity and phenolics in foods and dietary supplements. J. Agric. Food Chem. 2005, 53, 4290-4302. [CrossRef] [PubMed]

15. Zeb, A.; Ullah, S. Sea buckthorn seed oil protects against the oxidative stress produced by thermally oxidized lipids. Food Chem. 2015, 186, 6-12. [CrossRef] [PubMed]

16. Santos, J.; Oliveira, M.B.; Ibanez, E.; Herrero, M. Phenolic profile evolution of different ready-to-eat baby-leaf vegetables during storage. J. Chromatogr. A 2014, 1327, 118-131. [CrossRef] [PubMed]

17. Weisz, G.M.; Kammerer, D.R.; Carle, R. Identification and quantification of phenolic compounds from sunflower (Helianthus annuus L.) kernels and shells by HPLC-DAD/ESI-MSN. Food Chem. 2009, 115, 758-765. [CrossRef]

18. Aaby, K.; Ekeberg, D.; Skrede, G. Characterization of phenolic compounds in strawberry (Fragaria $\times$ ananassa) fruits by different HPLC detectors and contribution of individual compounds to total antioxidant capacity. J. Agric. Food Chem. 2007, 55, 4395-4406. [CrossRef] [PubMed]

19. Ruiz, D.; Egea, J.; Gil, M.I.; Tomas-Barberan, F.A. Characterization and quantitation of phenolic compounds in new apricot (Prunus armeniaca L.) varieties. J. Agric. Food Chem. 2005, 53, 9544-9552. [CrossRef] [PubMed]

20. Campbell, O.E.; Padilla-Zakour, O.I. Phenolic and carotenoid composition of canned peaches (Prunus persica) and apricots (Prunus armeniaca) as affected by variety and peeling. Food Res. Int. 2013, 54, 448-455. [CrossRef]

21. Dragovic-Uzelac, V.; Delonga, K.; Levaj, B.; Djakovic, S.; Pospisil, J. Phenolic profiles of raw apricots, pumpkins, and their purees in the evaluation of apricot nectar and jam authenticity. J. Agric. Food Chem. 2005, 53, 4836-4842. [CrossRef] [PubMed] 
22. Madrau, M.; Piscopo, A.; Sanguinetti, A.; Del Caro, A.; Poiana, M.; Romeo, F.; Piga, A. Effect of drying temperature on polyphenolic content and antioxidant activity of apricots. Eur. Res. Technol. 2009, 228, 441-448. [CrossRef]

23. Sultana, B.; Anwar, F. Flavonols (kaempeferol, quercetin, myricetin) contents of selected fruits, vegetables and medicinal plants. Food Chem. 2008, 108, 879-884. [CrossRef] [PubMed]

24. Razavi, S.; Zahri, S.; Zarrini, G.; Nazemiyeh, H.; Mohammadi, S. Biological activity of quercetin-3-O-glucoside, a known plant flavonoid. Russian J. Bioorgan. Chem. 2009, 35, 376-378. [CrossRef]

25. Morand, C.; Manach, C.; Crespy, V.; Remesy, C. Quercetin 3-O- $\beta$-glucoside is better absorbed than other quercetin forms and is not present in rat plasma. Free Radic. Res. 2000, 33, 667-676. [CrossRef] [PubMed]

26. Katayama, S.; Ogawa, H.; Nakamura, S. Apricot carotenoids possess potent anti-amyloidogenic activity in vitro. J. Agric. Food Chem. 2011, 59, 12691-12696. [CrossRef] [PubMed]

27. Katayama, T.; Nakayama, T.O.M.; Lee, T.H.; Chichester, C.O. Carotenoid transformations in ripening apricots and peaches. J. Food Sci. 1971, 36, 804-806. [CrossRef]

28. Sabir, A.; Ekbic, H.; Erdem, H.; Tangolar, S. Response of four grapevine (Vitis spp.) genotypes to direct or bicarbonate-induced iron deficiency. Span. J. Agric. Res. 2010, 8, 823-829. [CrossRef]

(C) 2017 by the authors; licensee MDPI, Basel, Switzerland. This article is an open access article distributed under the terms and conditions of the Creative Commons Attribution (CC BY) license (http://creativecommons.org/licenses/by/4.0/). 